\title{
Role of the Cdc25A phosphatase in human breast cancer
}

\author{
M. Giulia Cangi, ${ }^{1}$ Barry Cukor, ${ }^{1}$ Peggy Soung, ${ }^{1}$ Sabina Signoretti, ${ }^{1,2}$ Gilberto Moreira, Jr., ${ }^{1}$ \\ Moksha Ranashinge, ${ }^{1}$ Blake Cady, ${ }^{3}$ Michele Pagano, ${ }^{4}$ and Massimo Loda ${ }^{1,2}$ \\ ${ }^{1}$ Department of Adult Oncology, Dana Farber Cancer Institute, and \\ ${ }^{2}$ Department of Pathology, Brigham and Women's Hospital, Harvard Medical School, Boston, Massachusetts, USA \\ ${ }^{3}$ Department of Surgery, Brown University, School of Medicine, Providence, Rhode Island, USA \\ ${ }^{4}$ Department of Pathology, New York University Medical Center and Kaplan Comprehensive Cancer Center, New York, \\ New York, USA \\ Address correspondence to: Massimo Loda, Department of Adult Oncology, \\ Dana Farber Cancer Institute, 44 Binney Street, Dana 740B, Boston, Massachusetts 02215, USA. \\ Phone: (617) 632-4001; Fax: (617) 632-4005; E-mail: massimo_loda@dfci.harvard.edu.
}

Received for publication December 15, 1999, and accepted in revised form July 31, 2000.

The phosphatase Cdc25A plays an important role in cell cycle regulation by removing inhibitory phosphates from tyrosine and threonine residues of cyclin-dependent kinases, and it has been shown to transform diploid murine fibroblasts in cooperation with activated Ras. Here we show that Cdc25A is overexpressed in primary breast tumors and that such overexpression is correlated with higher levels of cyclin-dependent kinase 2 (Cdk2) enzymatic activity in vivo. Furthermore, in the breast cancer cell line MCF-7, Cdc25A activity is necessary for both the activation of Cdk2 and the subsequent induction of S-phase entry. Finally, in a series of small $(<1 \mathrm{~cm})$ breast carcinomas, overexpression of Cdc25A was found in $47 \%$ of patients and was associated with poor survival. These data suggest that overexpression of Cdc25A contributes to the biological behavior of primary breast tumors and that both Cdc25A and Cdk2 are suitable therapeutic targets in early-stage breast cancer.

J. Clin. Invest. 106:753-761 (2000).

\section{Introduction}

The key transitions in the process of eukaryotic cellular division are controlled by the sequential activation and inactivation of members of the cyclin-dependent kinase (Cdk) subfamily of protein kinases. The activities of these enzymes are regulated by multiple mechanisms including activating and inactivating phosphorylations, binding to regulatory cyclin subunits, subcellular localization, and association with Cdk inhibitors as well as controlled proteolysis of regulatory subunits (1-3).

Cyclin D-Cdk4/6 in mid-G1 and cyclin E-Cdk2 in late $\mathrm{G} 1$ are the Cdk complexes required for cellular progression past the restriction point, by committing the cells to division regardless of extracellular stimuli (4). p27 is the primary Cdk inhibitor responsible for inhibition of the cyclin E-Cdk2 complex. In contrast, the dual phosphatase Cdc25A is an integral component of cyclin E-Cdk2 activation (5).

Mammalian cells express at least three dual phosphatase Cdc25 homologues, named A, B, and C $(6,7)$. Although each of the three vertebrate $\mathrm{Cdc} 25$ proteins can dephosphorylate a variety of Cdk's in vitro, they are expressed and activated at different times during the cell cycle and have been proposed to act on different cyclin-Cdk complexes. Cdc25B and Cdc25C function primarily at the $\mathrm{G} 2 / \mathrm{M}$ transition, while $\mathrm{Cdc} 25 \mathrm{~A}$ promotes S-phase entry (7-12). In keeping with these roles, Cdc25A mRNA is expressed early in G1, with maximal levels occurring at the G1/S transition, while the Cdc25B mRNA peaks in G2 $(11,12)$.
The cyclin E-Cdk2 complex is phosphorylated on the Thr14 and Tyr15 residues of Cdk2 in vivo (9), and dephosphorylation is necessary for Cdk activation and S-phase initiation. Cdc25A inhibition through injection of anti-Cdc25A antibodies into both a normal rat kidney cell line (NRK) and human fibroblasts (IMR-90) prevents entry into $S$ phase, demonstrating that $\mathrm{Cdc} 25 \mathrm{~A}$ is required for cellular progression through the G1/S checkpoint $(10,12)$. Overexpression of Cdc25A has been shown to induce premature activation of both cyclin E- and cyclin A-Cdk2 complexes, without any demonstrable effect on cyclin D-dependent kinase (13). More recently, Cdc25A has been shown to be rapidly degraded by ubiquitin-proteasome-mediated proteolysis in response to ultraviolet light and ionizing radiation, resulting in a block in $S$ phase (14). Importantly, overexpression of Cdc25A eliminates this checkpoint $(14,15)$. Cdc25A and $\mathrm{B}$ cooperate with active Ras and with deletion of the $R B$ gene in transformation of murine fibroblasts (16). In addition, Cdc25B mRNA was found to be expressed at high levels in $32 \%$ of human breast cancers. Cdc25B overexpression was most frequently seen in high-histological-grade cancers and was associated with a decrease in disease-free survival at 10 years in patients who did not receive adjuvant therapy (ref. 16; M. Loda, unpublished data). More recently, Cdc25B was shown to induce mammary gland hyperplasia when the phosphatase was expressed as a transgene (17). 
Most of the data published on mammalian Cdc25A are based on experiments performed in fibroblasts. Here we present studies of the role of Cdc25A in a breast cancer cell line model and the evaluation of Cdc25A expression in a database of human breast cancers.

\section{Methods}

Patient population. This study was performed after approval by the Institutional Review Boards of the Dana-Farber Cancer Institute and of Brigham and Women's Hospital.

Archival $T_{1 a, b}$ breast carcinomas. A previously characterized series of breast carcinomas less than $1 \mathrm{~cm}$ in diameter $\left(\mathrm{T}_{1 \mathrm{a}, \mathrm{b}}\right)$ diagnosed between 1964 and 1994 was utilized (18). RNA preservation was adequate in 154 cases (18). In this study, a subset of 144 patients for whom tumor tissue was still available was analyzed by in situ hybridization with antisense riboprobes to Cdc25A. In this set of cases, p27 and Ki67 expression levels had been previously analyzed by immunohistochemistry (18). Population characteristics of this cohort have been previously published and are summarized in Table 1 . The mean age of the patients was 60.7 years, and the median follow-up was 66.4 months. Follow-up data were obtained from patients' charts and tumor registry records. Death from cancer was accepted when confirmed by autopsy or when there was convincing evidence of disease by clinical, pathologic, or radiographic parameters. Table 2 is a summary of the adjuvant therapy received in a subset of patients $(n=61)$.

Frozen breast carcinomas. Since no frozen tissue was available from any of the patients with $T_{1 a, b}$ cancers, 21 frozen tissue samples of breast carcinoma were randomly selected from a series of over 100 patients who were operated upon between 1989 and 1994. Tumor specimens were characterized in terms of pathologic variables, including tumor size $\left(13 \mathrm{~T}_{1 \mathrm{c}}, 7 \mathrm{~T}_{2}\right.$, and $\left.1 \mathrm{~T}_{3}\right)$ and histologic grade ( 9 grade II and 12 grade III). For each case a hematoxylin and eosin-stained frozen section was prepared to ensure the presence of tumor in the samples. Nontumor tissue surrounding the infiltrating cancer was dissected away so that at least $80 \%$ of each sample used for RNA and protein extraction was made up of carcinoma. Ten cases of normal breast tissue obtained from reduction mammoplasty specimens were utilized as control.

In situ bybridization. $1 \mu \mathrm{g}$ of recombinant plasmid pBluescript II (Stratagene, La Jolla, California, USA) containing the 514-bp 5' end of the human Cdc25A gene from the ATG start codon was linearized using BamHI and SalI restriction enzymes (Life Technologies Inc., Gaithersburg, Maryland, USA) to generate sense and antisense transcripts, respectively. Digoxigeninlabeled riboprobes were used. In situ hybridization was performed on the automated in situ hybridization instrument Gen II (Ventana Medical Systems, Tucson, Arizona, USA) using identical incubation and detection time as previously described, with minor modifications (hybridization at $60^{\circ} \mathrm{C}$ for 3 hours) $(16,19)$.
For the scoring of Cdc25A, a total of 1000 cells per case was counted independently by two investigators. Only tumor cells strongly positive for Cdc25A were counted. Categories were made on the basis of percent positive cells: $0=$ negative cases, $1=0-25 \%$ positive cells, $2=25-50 \%, 3>50 \%$. Cases were deemed to be overexpressers only when more than $50 \%$ of cells expressed high levels of Cdc25A (category 3). Patients were thus subdivided on the basis of expression as low expressers or nonexpressers (categories 0,1 , and 2 ) versus overexpressers (category 3) as previously described (16) (Table 1). All statistical analyses were performed comparing these two groups.

Immunoprecipitation, immunoblotting, and kinase assays. Frozen breast tumors were thawed and lysed in $200 \mu \mathrm{L}$ of lysis buffer (10\% sucrose, $20 \mathrm{mM}$ Tris [pH 8.0], 137 $\mathrm{mM} \mathrm{NaCl}, 10 \%$ glycerol, 2 mM EDTA, $10 \mathrm{mM} \mathrm{NaF}, 1$ $\mathrm{mM} \mathrm{Na}_{3} \mathrm{VO}_{4}, 1 \mathrm{mM}$ PMSF, $1 \%$ Nonidet P-40 substitute [Fluka, Ronkonkoma, New York, USA], and $1 \mu \mathrm{g} / \mathrm{mL}$ leupeptin). MCF-7 cells before and after transfection with antisense or sense Cdc25A oligonucleotides were lysed in RIPA buffer (1\% Nonidet P-40 substitute [Fluka], 1\% sodium deoxycholate, $0.1 \%$ SDS, $0.15 \mathrm{M}$ $\mathrm{NaCl}, 0.01 \mathrm{M}$ sodium phosphate, $2 \mathrm{mM}$ EDTA, $50 \mathrm{mM}$ sodium fluoride, and $0.2 \mathrm{mM}$ sodium vanadate) containing $100 \mathrm{mM}$ PMSF, $100 \mathrm{mM}$ DTT, and protease inhibitor cocktail (Boehringer Mannheim Biochemicals Inc., Indianapolis, Indiana, USA).

Conditions for immunoprecipitation and immunoblotting have been previously described (20). For immunoprecipitation, Cdk 2 and Cdk4 rabbit polyclonal (Santa Cruz Biotechnology Inc., Santa Cruz, California, USA), and p27 mouse monoclonal (Transduction Laboratories, Lexington, Kentucky, USA) antibodies were used. For immunoblotting, cyclin E $(2.5 \mu \mathrm{g} / \mathrm{mL}$; Oncogene Research Products, Cambridge, Massachusetts, USA), Cdk2 (1 $\mu \mathrm{g} / \mathrm{mL}$; Santa Cruz Biotechnology Inc.), p27 (0.1 $\mu \mathrm{g} / \mathrm{mL}$; Transduction Laboratories), phosphotyrosine (2 $\mu \mathrm{g} / \mathrm{mL}$; Upstate Biotechnology Inc., Lake Placid, New York, USA), and Cdc25A (1 $\mu \mathrm{g} / \mathrm{mL}$; Santa Cruz Technology) antibodies were used. Densitometric analysis was performed on the developed Western blot for Cdc25A. To normalize the values, a ratio of the percent area of the tested samples to the one obtained with the control not treated by antisense oligonucleotides was used.

$\mathrm{Cdk} 2$ histone $\mathrm{H} 1$ kinase assay was performed as previously described (21). Cdk4 kinase assay was performed essentially with the same methodology, substituting histone $\mathrm{H} 1$ with $5 \mathrm{mg} / \mathrm{mL}$ of $\mathrm{Rb}$ peptide (Santa Cruz Biotechnology Inc.) as substrate. HeLa cell lysate was used as a positive control. Densitometric analysis was performed on the developed autoradiography. To normalize the values, a ratio of the percent area of the tested samples to the one obtained with the HeLa control in each gel was used. To account for variability from gel to gel, this ratio was, in turn, related (as a ratio) to the total area under all curves in a given gel. Normalized values were used in the statistical analysis. 
Semiquantitative RT-PCR for Cdc25A levels. Frozen tissue samples were ground by a tissue homogenizer, and both frozen tissue and cell line RNA were extracted using a guanidine isothiocyanate protocol (TRI Reagent; Molecular Research Center Inc., Cincinnati, Ohio, USA). Cdc25A cDNA was synthesized from equal amounts of RNA $(1 \mu \mathrm{g})$ with the downstream primer, 5'-CCGGTAGCTAGGGGGCTCACA-3', encompassing the catalytic domain, using murine leukemia virus reverse transcriptase $(2.5 \mathrm{U} / \mu \mathrm{L})$ (Perkin-Elmer Corp., Norwalk, Connecticut, USA). PCR was performed in $25-\mu \mathrm{L}$ reaction mixture containing DNA template, PCR buffer $(50 \mathrm{mM} \mathrm{KCl}, 10 \mathrm{mM}$ Tris- $\mathrm{HCl}$, $\mathrm{pH}$ 8.3), $1.5 \mathrm{mM} \mathrm{MgCl}_{2}, 200 \mu \mathrm{M}$ of each dNTP, 0.4 $\mu \mathrm{M}$ of each primer (upstream primer $5^{\prime}$-AGCCCCAAAGAGTCAACTAATCCAGA-3'), and 0.75 units of AmpliTaq DNA polymerase (Perkin-Elmer Corp.) to amplify a 570-bp product. PCR was performed for 35 cycles consisting of $95^{\circ} \mathrm{C}$ for 1 minute, $63^{\circ} \mathrm{C}$ for 2 minutes, and $72^{\circ} \mathrm{C}$ for 1 minute. Levels of $\mathrm{Cdc} 25 \mathrm{~A}$ were compared with those of the small trimeric $\mathrm{G}$ protein $\mathrm{Gs} \alpha$ (upstream primer 5'-GTGATCAAGCAGGCTGACTAT-3' downstream primer 5'-GCTGCTGGCCACCACGAAGATGAT-3'), which was reverse-transcribed and amplified (200-bp product) using the same conditions described above for Cdc25A. The resulting amplification products were then analyzed by agarose gel electrophoresis using standard methods, and densitometry was performed.

For statistical analysis, the ratio of the densitometric values of Cdc25A to those of Gs $\alpha$ was calculated and the median value (median $=1$ ) was utilized as a cutoff for the categorization of cases into high and non-/low expressers. A sample was considered a high expresser for $\mathrm{Cdc} 25 \mathrm{~A}$ when this ratio was $\geq 1$; non-/low expressers showed a ratio $<1$.

Cdc25A antisense oligonucleotide transfection. The breast carcinoma cell line MCF7 (American Type Culture Collection, Rockville, Maryland, USA) was maintained in DMEM supplemented with $10 \%$ FBS (Life Technologies Inc.). MCF-7 cells, $60 \%$ confluent, were transfected with antisense (TCAAACACAAACACGACT) or scrambled (ATCCGAACTCAACAAACA) oligonucleotides (New Second Generation Chimeras, Oligos Etc. Inc., Bethel, Maine, USA) for Cdc25A according to Life Technologies Inc. directions. MCF-7 cells were incubated with a DNA-lipid solution consisting of 400-nM oligonucleotides, $12 \mu \mathrm{L}$ of lipofectin reagent (Life Technologies Inc.) and Opti-MEM I reduced serum media (Life Technologies Inc.) for 6 hours at $37^{\circ} \mathrm{C}$. Cells were harvested 24 hours and 48 hours after transfection.

Antisense experiments were also performed cotransfecting (a) the $\mathrm{Cdk} 2 \mathrm{AF}$

\section{Table 1}

mutant $(7.8 \mu \mathrm{g})$, in which the phosphorylatable sites Thr14 and Tyr15 were replaced with nonphosphorylatable residues (Ala14 and Phe15); and (b) wild-type Cdk2 $(10 \mu \mathrm{g})$. Cdk2AF cannot be phosphorylated in the critical residues normally dephosphorylated by Cdc25A phosphatase (22).

Proliferation and cell cycle analysis. Cells were pulselabeled with $10 \mathrm{mM}$ bromodeoxyuridine (BrdU) at $37^{\circ} \mathrm{C}$ for 30 minutes, harvested, fixed in $70 \%$ ethanol, and treated with $0.2 \mathrm{~N} \mathrm{HCl}$ for 30 minutes. Cells were then stained with anti-BrdU FITC-conjugated (Boehringer Mannheim Biochemicals Inc.) and counterstained with propidium iodide $(5 \mu \mathrm{g} / \mathrm{mL})$. Flow cytometric analysis was performed using a Becton Dickinson FACScan flow cytometer (Becton Dickinson and Co., Franklin Lakes, New Jersey, USA).

Statistical analysis. In the archival $\mathrm{T}_{1 \mathrm{a}, \mathrm{b}}$ breast carcinomas, the primary study outcome was disease-free survival, which was measured from the date of surgery to the date of last follow-up or death. Survival was censored if the patient was still alive or died from other causes. Survival curves for Cdc25A and p27 were constructed using the Kaplan-Meier method. Univariate survival curves were compared using a Wilcoxon procedure, and differences between prognostic factors were tested for statistical significance with the Logrank analysis. A $P$ value of less than 0.05 was required for significance. Fisher's exact test was used to test for an association between Cdc25A and Ki67 expression levels and to compare patients who received adjuvant treatment with those

Clinicopathological features of study population in relation to $\mathrm{Cdc} 25 \mathrm{~A}$ status

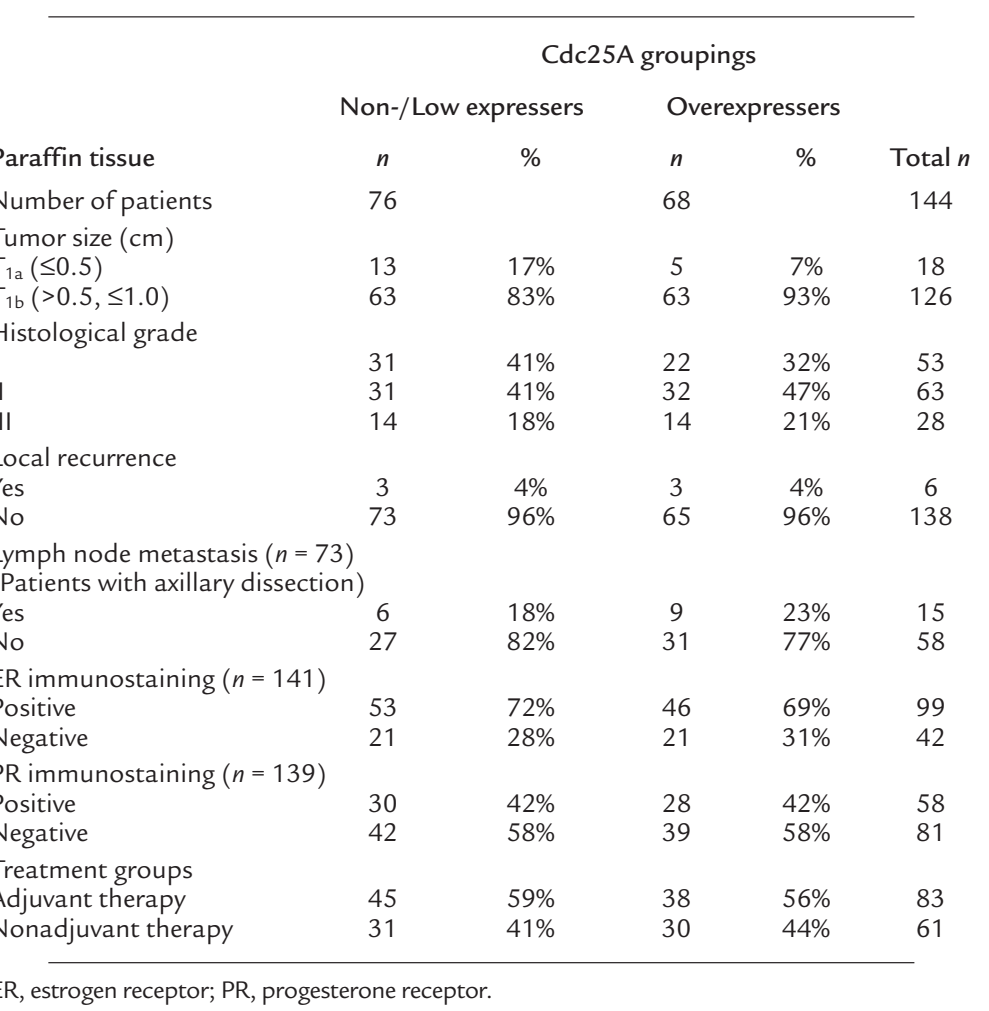


Table 2

Methods of treatment versus p27 and Cdc25A levels in the tumors

\begin{tabular}{|c|c|c|c|c|c|c|c|c|c|c|c|c|c|c|c|c|c|}
\hline \multirow[b]{3}{*}{$\mathrm{p} 27$} & \multirow[b]{3}{*}{ Cdc25A } & \multicolumn{16}{|c|}{ Methods of treatment } \\
\hline & & \multicolumn{2}{|c|}{ RT } & \multicolumn{2}{|c|}{ CT } & \multicolumn{2}{|c|}{ TAM } & \multicolumn{2}{|c|}{$\mathrm{RT}+\mathrm{CT}$} & \multicolumn{2}{|c|}{$\mathrm{CT}+\mathrm{TAM}$} & \multicolumn{2}{|c|}{$\mathrm{RT}+\mathrm{TAM}$} & \multicolumn{2}{|c|}{$\mathrm{RT}+\mathrm{CT}+\mathrm{TAM}$} & \multicolumn{2}{|c|}{ None } \\
\hline & & $n$ & $\%$ & $n$ & $\%$ & $n$ & $\%$ & $n$ & $\%$ & $n$ & $\%$ & $n$ & $\%$ & $n$ & $\%$ & $n$ & $\%$ \\
\hline+ & + & 8 & 20 & 1 & 2.5 & 3 & 7.5 & 3 & 7.5 & 0 & 0 & 5 & 12.5 & 1 & 2.5 & 19 & 47.5 \\
\hline+ & - & 10 & 29.4 & 0 & 0 & 4 & 11.8 & 0 & 0 & 0 & 0 & 1 & 2.9 & 0 & 0 & 19 & 55.9 \\
\hline - & + & 7 & 25 & 1 & 3.6 & 3 & 10.7 & 4 & 14.3 & 0 & 0 & 1 & 3.6 & 1 & 3.6 & 11 & 39.3 \\
\hline - & - & 14 & 33.3 & 0 & 0 & 6 & 14.3 & 2 & 4.8 & 0 & 0 & 7 & 16.7 & 1 & 2.4 & 12 & 28.6 \\
\hline \multicolumn{2}{|c|}{ Total number of patients } & 39 & & 2 & & 16 & & 9 & & 0 & & 14 & & 3 & & 61 & \\
\hline
\end{tabular}

$\mathrm{RT}$, radiation therapy; $\mathrm{CT}$, chemotherapy; TAM, tamoxifen; None, none of the stated treatments were used.

who did not in the four categories stratified according to $\mathrm{Cdc} 25 \mathrm{~A}$ and $\mathrm{p} 27$ status.

All data obtained from the frozen breast cancer tissues were analyzed by a linear regression model. This was used to explain Cdk2 enzymatic activity in terms of p27 (before or after immunoprecipitation), Cdk2, cyclin E, and Cdc25A levels.

\section{Results}

Cdc25A is overexpressed in breast cancer. In order to determine whether Cdc25A was overexpressed in breast carcinomas, we first used a series of 21 breast carcinomas in which frozen tissues were available. In addition, as controls, ten samples of normal breast tissue obtained from reduction mammoplasties were analyzed in parallel. After enrichment of tumor cells to at least $80 \%$ by macrodissection, RNA extracted from these tissues was subjected to RT-PCR with primers specific to $C D C 25 \mathrm{~A}$ and, as an amplification control, primers specific for the small trimeric G-protein Gs $\alpha$. Amplified products were separated by agarose gel electrophoresis and quantitated by densitometry. In normal breast tissue there is little to no detectable Cdc25A mRNA (data not shown). In contrast, 11 of the 21 breast carcinoma samples (52\%) were found to express high levels of Cdc25A, defined by a ratio of Cdc25A to Gs $\alpha$ of $\geq 1$. Representative high and low expressers of Cdc25A are shown in Figure 1a.

In addition, $\mathrm{Cdc} 25 \mathrm{~A}$ expression was determined in a series of 144 formalin-fixed, paraffin-embedded $T_{1 a, b}$ breast carcinomas and adjacent normal tissues by in situ hybridization with antisense and, as a control, sense CDC25A riboprobes (Figure 2). Tables 1 and 2 show the pathologic characteristics and therapeutic regimens of this series of carcinomas. In agreement with the data obtained by RT-PCR, faint or no expression of Cdc25A was detectable in normal $(\mathrm{N})$ breast tissue (Figure $2 \mathrm{~b}$ and $\mathrm{c}$ ), while high expression was found in $47 \%$ of primary tumors $(\mathrm{T})$ when compared with the adjacent normal breast epithelium (Table 1; Figure 2, b, e, and f).

Increased expression of $C d c 25 \mathrm{~A}$ is associated with $\mathrm{Cdk} 2$ activation in human breast cancers. Cdk2 kinase activity is regulated by Cdc25A-mediated dephosphorylation of Cdk2 at residues threonine 14 and tyrosine 15 . There- fore, we asked whether elevated levels of Cdc25A in the 21 primary breast tumors resulted in or were associated with a change in Cdk2 kinase activity. To this end, lysates were prepared from normal breast and breast carcinoma tissue, and Cdk2 was immunoprecipitated and used for in vitro kinase reactions using histone $\mathrm{H} 1$ as a substrate. Cdk2 activity is governed positively by the levels of associated cyclin $\mathrm{E}$ and negatively by associated $\mathrm{p} 27$. Thus, in parallel, we also determined the levels of p27 (total and after immunoprecipitation with Cdk2), Cdk2, and cyclin E protein by Western blot (Figure 1 and data not shown). a
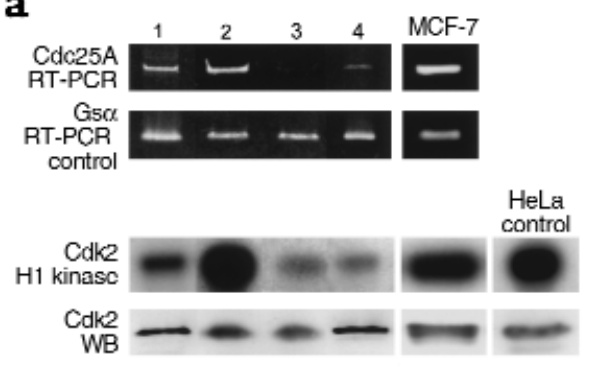

b

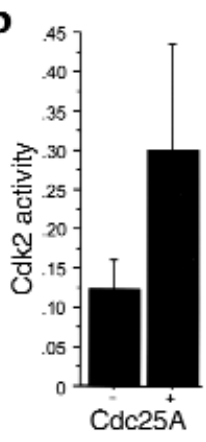

\section{Figure 1}

Cdc25A expression levels are directly correlated with Cdk2 activity. (a) Cdc25A and Gs $\alpha$ mRNA levels were determined by semiquantitative RT-PCR analysis (upper panel) on microdissected frozen tissue samples of breast carcinomas and on MCF-7 cells. Four representative cases of high expressers (lanes 1 and 2 ) and low expressers (lanes 3 and 4) for Cdc25A are shown. Cdk2 enzymatic activity and Cdk2 protein level for the four tissue samples of breast carcinoma (lanes 1-4), for MCF-7 cells, and for the control sample HeLa cells are shown in the lower panel. A direct correlation between Cdc25A levels and Cdk2 enzymatic activity is evident, whereas Cdk2 levels remain unchanged. (b) Bar graph representing cumulative data of Cdc25A levels compared with Cdk2 enzymatic activity in 21 patients. The ratio of the densitometric values of $\mathrm{Cdc} 25 \mathrm{~A}$ to those of $\mathrm{Gs} \alpha$ was calculated, and the median value was used as a cutoff for the categorization of cases into high and low/nonexpressers. High expressers $(+)$ and low/nonexpressers (-) for Cdc25A were correlated with the corresponding Cdk2 enzymatic activity. To normalize densitometric values of Cdk2 activity, a ratio of the percent area of the tested samples to the one obtained with the HeLa control in each gel was used. To account for variability from gel to gel, this ratio was, in turn, related as a ratio to the total area under all curves in a given gel. 
No appreciable Cdk2 enzymatic activity was detectable in any of the lysates from normal breast tissue obtained from reduction mammoplasty specimens, in spite of normal levels of C $\mathrm{dk} 2$ by Western blot. In contrast, primary breast tumors were found to have markedly variable levels of Cdk2 kinase activity, despite an absence of variability in the levels of Cdk2 protein. Furthermore, the levels of Cdk2 protein were comparable with those found in normal breast tissue. While differences in $\mathrm{Cdk} 2$ protein levels failed to account for intertumor variability in Cdk2 kinase activity, a direct correlation between Cdk2 activity and Cdc25A expression was found in the 21 breast carcinomas examined (Figure $1 \mathrm{~b}$ ). In addition, high cyclin $\mathrm{E}(P=0.005)$ and $\mathrm{Cdc} 25 \mathrm{~A}$ mRNA $(P=0.008)$ levels were significant predictors of Cdk2 activity in a linear regression model. The overall $r^{2}$ for the model was 0.58 . Thus $58 \%$ of the total variation in Cdk2 kinase activity found in the breast tumors was explained by the levels of Cdc25A mRNA and cyclin E protein. A trend of inverse correlation between Cdk2 activity and complex-bound p27 (but not total p27) levels was found, although this was not statistically significant.

Cdc25A is required for full activation of Cdk2. These data, together with those in the literature, suggest that $\mathrm{Cdc} 25 \mathrm{~A}$ is a major regulator of Cdk2 kinase activity and raised the possibility that in breast cancers that express high levels of Cdc25A this phosphatase may be required for the maintenance of Cdk 2 activity. In order to test this hypothesis, MCF-7 breast carcinoma cells were used as a model of a cell line that expresses high levels of Cdc25A (Figure 1a). MCF-7 cells were transfected with antisense or scrambled oligonucleotides for CDC25A. Untransfected proliferating cells were also used as control. Transfection with antisense, but not scrambled, oligonucleotides led to a marked decrease in Cdc25A (but not Gs $\alpha$ ) mRNA levels at the 24- and 48-hour time points (Figure 3a). Similarly, protein levels of Cdc25A were diminished after antisense transfection as assessed by Western blot (Figure 3a). In spite of unchanged levels of Cdk 2 detected by Western blot, Cdk2 enzymatic activity decreased by $73 \%$ at 24 hours and by $88 \%$ at 48 hours after antisense transfection, while Cdk4 activity was unaffected (Figure $3 b$ ).

Cdc25A can dephosphorylate tyrosine 15 of Cdk2 leading to kinase activation. We thus asked whether, in antisense transfected cells, tyrosine phosphorylation of $\mathrm{Cdk} 2$ was altered. Following antisense transfection, immunoprecipitated Cdk2 was blotted with phosphotyrosine, cyclin E, p27, and Cdk2 antibodies. Antisense transfection resulted in a marked increase in Cdk2 tyrosine phosphorylation compared with both untransfected cells and those transfected with scrambled oligonucleotides. Somehow, surprisingly, antiCdk2 immunoprecipitates from antisense transfected cells were also found to have a marked reduction in coimmunoprecipitated levels of p27 but not cyclin E (Figure 3c). The decrease in associated p27 was not sim-
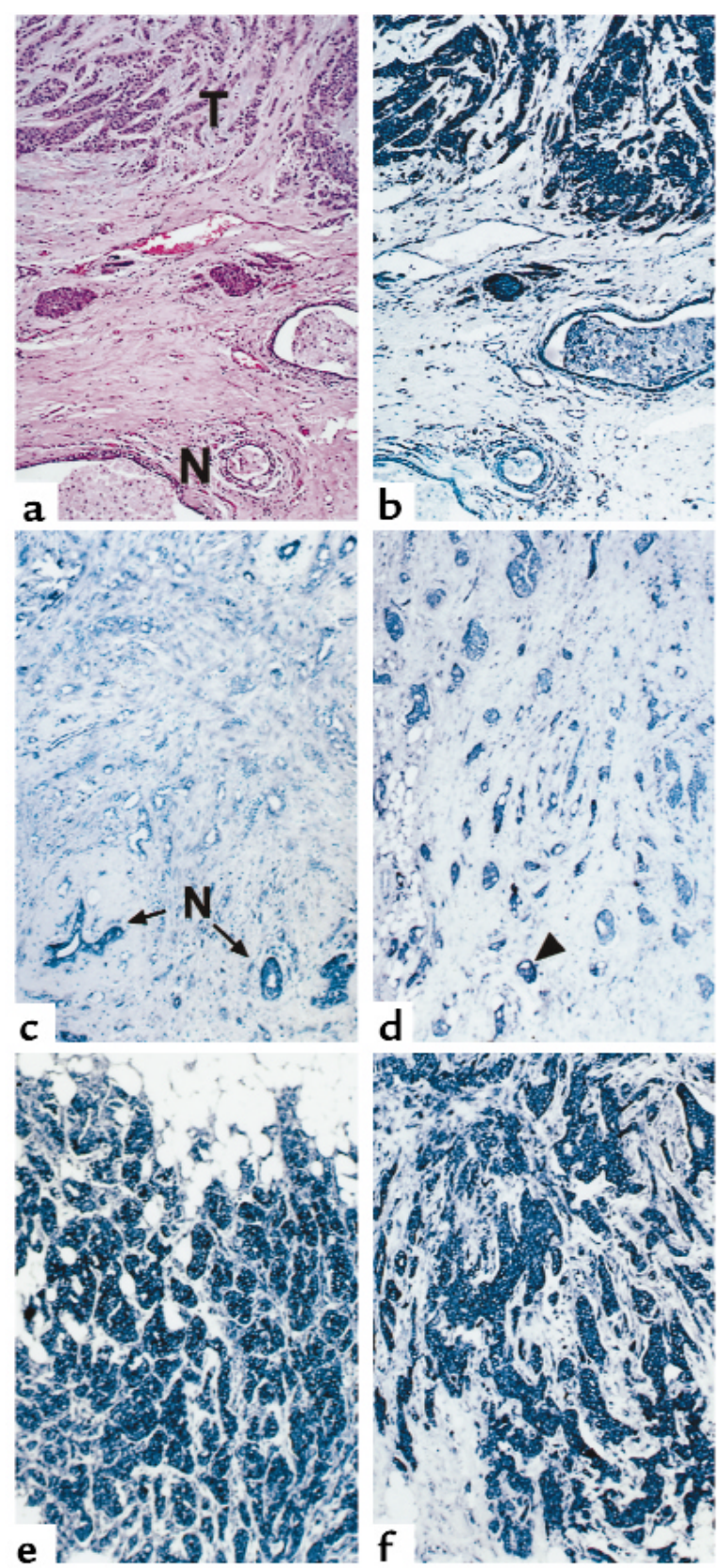

Figure 2

Cdc25A is overexpressed in human breast cancer. (a) Hematoxylin-and-eosin stain of invasive ductal carcinoma of the breast (T). Normal ducts are seen in the lower left corner ( $N)$. (b) In situ hybridization with digoxigenin-labeled, antisense riboprobe for Cdc25A demonstrates high expression levels of the phosphatase in tumor cells. Scant to no expression is seen in the adjacent normal breast tissue. (c) Representative example of a nonexpresser: no expression of $C d c 25 \mathrm{~A}$ is seen in the infiltrating ductal carcinoma cells. Scattered residual normal ducts $(\mathrm{N})$ show weak expression of the phosphatase. (d) Representative example of a low expresser for Cdc25A: most infiltrating ductal carcinoma cells do not express Cdc25A mRNA. Rare ducts (arrowhead) show weak expression of the phosphatase. (e and $\mathbf{f}$ ) Representative examples of high expressers for cdc25A: all infiltrating ductal carcinoma cells show strong staining with the antisense riboprobe for Cdc25A (Panels b, $\mathbf{c}, \mathbf{d}, \mathbf{e}$, and $\mathbf{f}$ : alkaline phosphatase: nitro blue tetrazolium cytoplasmic reaction product, section counterstained with methyl green). Sense controls were negative (not shown). 
a

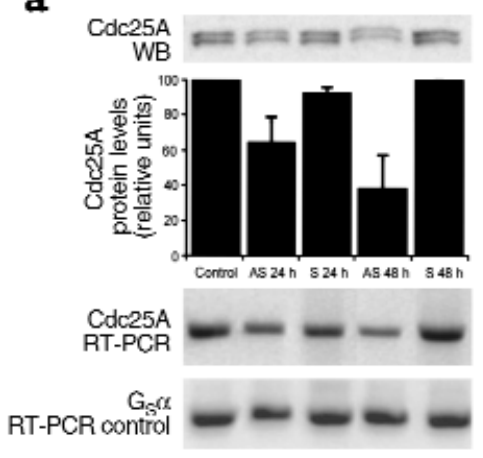

b
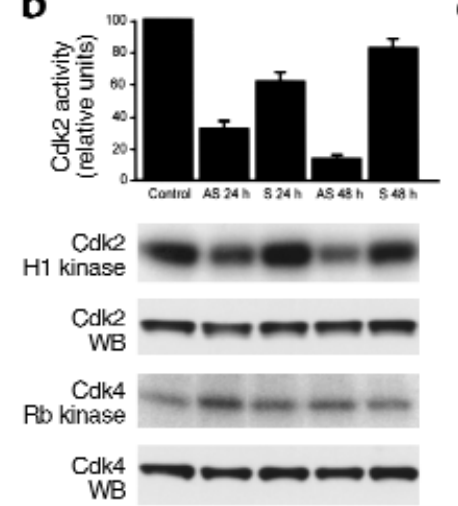

C
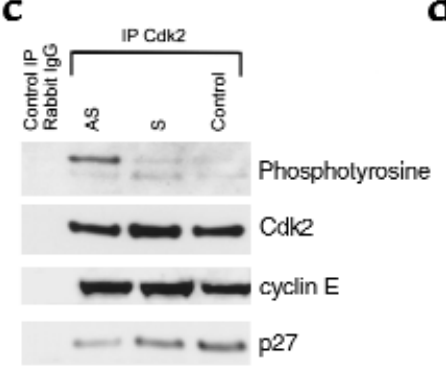

d

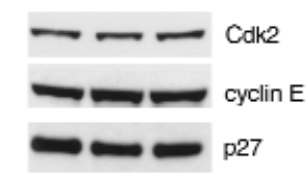

\section{Figure 3}

( $\mathbf{a}$ and $\mathbf{b}$ ) Antisense oligonucleotides directed to Cdc25A decrease Cdk2 activity and inhibit S-phase entry in MCF-7 cells. MCF-7 cells transfected with antisense (AS) or scrambled (S) oligonucleotides for Cdc25A were analyzed at 24 hours and 48 hours after transfection. Untransfected, proliferating MCF-7 cells represent base-line values (Control). (a) Immunoblot and RT-PCR for Cdc25A show significant decreases in Cdc25A protein and mRNA levels at 24 hours and 48 hours after transfection with AS but not $\mathrm{S}$ oligonucleotides. The bar graph is the densitometric quantitation of Cdc25A protein levels in three separate experiments. A $61 \%$ decrease in Cdc25A protein level was noted at 48 hours following AS transfection. No change is noted in Gs $\alpha$ mRNA levels after transfection. (b) Histone H1 and $\mathrm{Rb}$ kinases for $\mathrm{Cdk} 2$ and $\mathrm{Cdk} 4$ after immunoprecipitation with the respective antibodies and corresponding immunoblot. Cdk2 enzymatic activity decreases by $73 \%$ at 24 hours and by $88 \%$ at 48 hours after AS but not S transfection. In contrast, Cdk4 activity remains unchanged. No change in either Cdk2 or Cdk4 protein levels (Cdk2 WB and Cdk4 WB) is seen. WB, Western blot. (c and d) Antisense oligonucleotides against $C d c 25 A$ result in increased $C d k 2$ phosphorylation and decreased complexed p27. MCF-7 cells were transfected with antisense (AS) or scrambled (S) CDC25A oligonucleotides and analyzed 48 hours after transfection. Untransfected proliferating MCF-7 cells represent base-line values (Control). (c) Immunoprecipitated lysates with unrelated rabbit lgG (left) and Cdk2 were blotted with phosphotyrosine, Cdk2, cyclin E, and p27 antibodies. AS CDC25A results in a marked increase in Cdk2 phosphorylation. Concomitant to this, a decrease in complexed p27 is seen. (d) Total lysate immunoblots for Cdk2, cyclin E, and p27. No change in total levels of Cdk2, cyclin E, and p27 is seen.

ply a reflection in alterations in total $\mathrm{p} 27$, as no change in total levels of Cdk2, cyclin E, and p27 were detectable after antisense transfection (Figure 3d). Thus, inhibition of Cdc25A and the subsequent reconstitution of tyrosine phosphorylation of Cdk2 lead to a marked reduction in Cdk2 kinase activity despite a decrease in the association of the inhibitor $\mathrm{p} 27$. These data strongly suggest that $\mathrm{Cdc} 25 \mathrm{~A}$ is required for maintaining Cdk2 kinase activity in these cells.

Inactivation of Cdc25A inhibits S-phase entry in breast carcinoma cells. We next sought to determine whether inactivation of Cdc25A had functional consequences on cell cycle progression. MCF-7 cells were again transfected with antisense or scrambled oligonucleotides to CDC25A. The cell-cycle distribution of the entire population of cells was determined by BrdU labeling and two-color FACS analysis utilizing an FITC-conjugated anti-BrdU antibody and propidium iodide to measure DNA content. In these experiments, transfection of antisense oligonucleotides, but not sense oligonucleotides, resulted in a marked inhibition of S-phase entry (Figure 4, a and b). Inhibition of S-phase entry by antisense treatment was completely reversed by cotransfection of a constitutively active form of Cdk2 (Figure 4, a and b). This mutant form of Cdk2 (Cdk2AF) contains substitutions at threonine 14 and tyrosine 15 that render the protein insensitive to inactivating phosphorylations and is thus no longer regulated by Cdc25A. Importantly, transfection of the antisense oligonucleotide to Cdc25A with wild-type Cdk2 resulted in a decrease in Cdk2 activity, strongly suggesting that the activating dephosphorylations of $\mathrm{Cdc} 25 \mathrm{~A}$ are required for $\mathrm{Cdk} 2$ activity and S-phase entry in MCF-7 cells. Together the above data support the notion that in these cells Cdc25A-mediated dephosphorylation of Cdk2 is required for cell cycle progression.

High Cdc25A expression is associated with poor survival in patients with $T_{1 a, b}$ breast carcinoma. Since we had established that Cdc25A is overexpressed in breast carcinomas and that overexpression of this phosphatase was positively associated with Cdk 2 enzymatic activity and cell cycle progression, we examined whether overexpression of Cdc25A was associated with outcome in the cohort of patients with breast carcinomas $\leq 1$ centimeter in diameter $\left(\mathrm{T}_{1 \mathrm{a}, \mathrm{b}}\right)$.

Cdc25A levels were determined by in situ hybridization in 144 cases of breast carcinomas $\leq 1$ centimeter in diameter $\left(T_{1 a, b}\right)$. Patients with tumors overexpressing $\mathrm{Cdc} 25 \mathrm{~A}$ had an increased risk of dying of disease compared with nonexpressers by univariate analysis $($ Logrank $=0.0198$, Wilcoxon $=0.0231)($ Figure 5a). In fact, the group of patients with overexpression of Cdc25A had $24 \%$ mortality compared with $5 \%$ in patients who did not express Cdc25A. When the original cohort of 202 patients with $T_{1 a, b}$ breast cancer was analyzed, p27 was found to have independent prognostic significance, with low expression predicting 


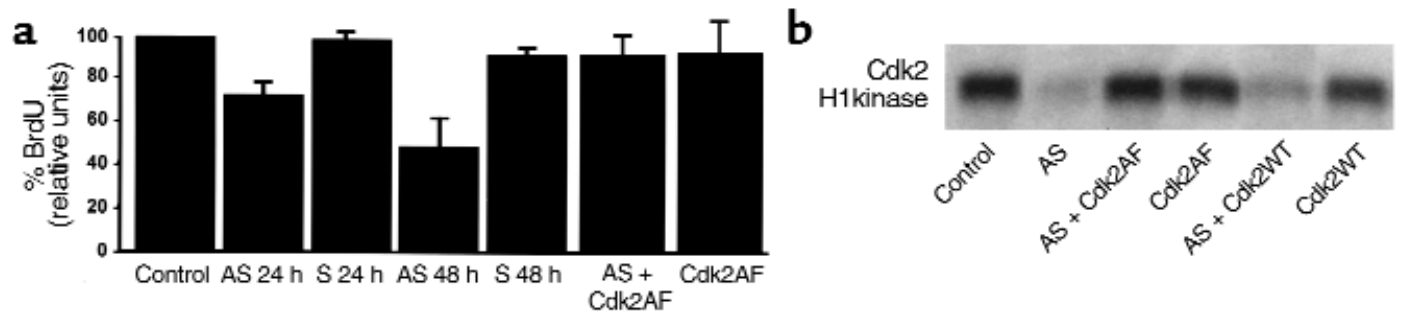

\section{Figure 4}

Inactivation of Cdc25A inhibits S-phase entry in breast carcinoma cells. (a) S phase in MCF-7 cells is measured by FACS analysis of BrdU-stained cells. Antisense oligonucleotides inhibit S-phase entry by $31 \%$ at 24 hours and $43 \%$ at 48 hours after AS transfection. No change is noted after transfection with scrambled oligonucleotides. S-phase entry is restored by the cotransfection of the nonphosphorylatable mutant of Cdk2, Cdk2AF, and antisense oligonucleotides to Cdc25A. (b) Nonphosphorylatable Cdk2 catalytic mutant (Cdk2AF) abolishes Cdc25A antisense effects on MCF-7. MCF-7 cells were transfected with antisense Cdc25A (AS), wild-type Cdk2 (Cdk2WT), or Cdk2AF, or cotransfected with AS and Cdk2AF or AS and Cdk2WT. Untransfected proliferating MCF-7 cells represent base-line values of Cdk2 activity and S-phase fraction (Control). At 48 hours after transfection, Cdk2 kinase activity (a) and BrdU incorporation as a measure of S-phase fraction (b) show that Cdk2AF, but not Cdk2WT, abolishes the Cdc25A AS effect.

poor survival, while Ki67 had no predictive value of survival status (18). In the residual 144 cases used here, stratification according to p27 status showed a trend toward predicted survival but was not significant. A stratification of mortality risk was identified when four different combinations of Cdc25A and p27 levels were evaluated for their relative effect on survival. The group with the highest mortality was the one in which breast carcinomas overexpressed Cdc25A and had low (<50\% of cells) p27 levels $($ Logrank $=0.0218$, Wilcoxon $=0.0233)($ Figure $5 b)$. Interestingly, the two curves with the worse survival (red and black curves) represented patients with overexpression of Cdc25A regardless of $\mathrm{p} 27$ status, rather than patients with low p27 (green and black curves). When a Cox proportional hazards model was constructed for the entire series, nodal status was the only significant covariate $(P=0.0010)$; age, tumor differentiation, extent of intraductal component, tumor size, c-erbB2, p53, p27, estrogen receptor- and prog- esterone receptor-immunostaining, $\mathrm{Cdc} 25 \mathrm{~A}(P=$ 0.1241 ), and Cdc25B were not significant covariates. There was no statistically significant difference when we compared patients who received adjuvant treatment with those who did not in the four categories stratified according to Cdc25A and p27 status $(P=$ $0.10)$ (Table 2). Ki67 had no prognostic significance, and no association was found between $\mathrm{Cdc} 25 \mathrm{~A}$ and Ki67 expression levels $(P=0.40)$.

When patients with axillary dissection were analyzed as a group $(n=73)$, the presence of lymph node metastases was a significant predictor of behavior by univariate analysis $(P<0.001)$. In this group Cdc25A still showed a trend toward discrimination of biologic behavior, although this was not statistically significant $(P<0.09)$. Interestingly however, in the 15 patients with lymph node metastasis, seven of nine patients with concomitant overexpression of Cdc25A died of disease. In contrast, none of the six with Cdc25A-negative tumors died. a

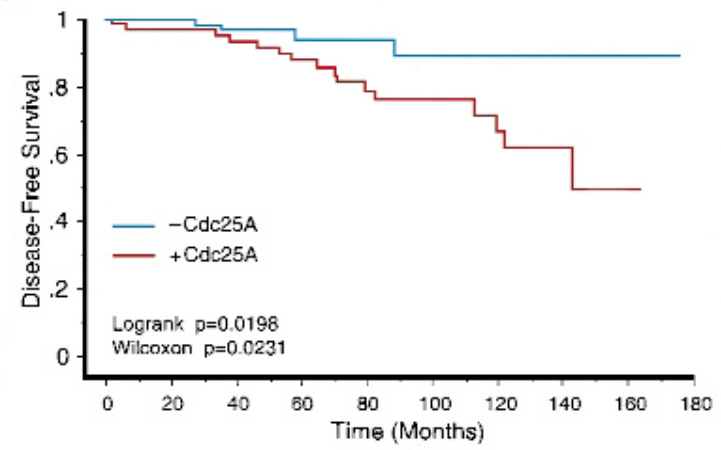

b

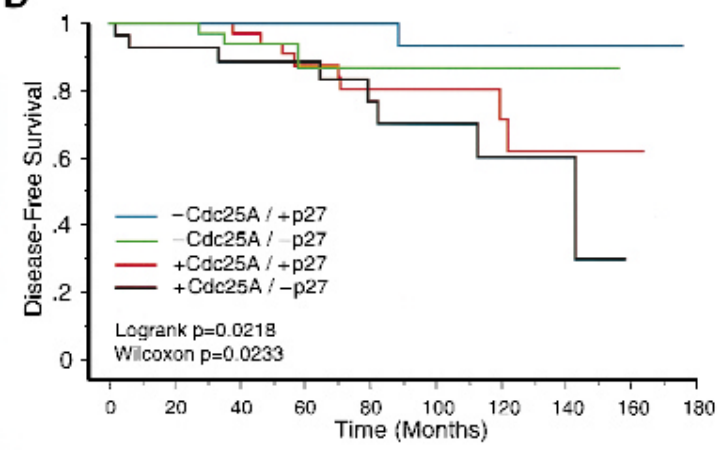

Figure 5

Cdc25A overexpression, with or without concomitant loss of p27, is associated with poor survival. (a) High levels of Cdc25A are associated with decreased disease-free survival in the 144 patients studied. (b) When Cdc25A is analyzed together with p27, stratification of disease-related mortality risk is based on four different combinations of Cdc25A and p27 levels. The group with the highest mortality is the one in which breast carcinomas overexpress Cdc25A and have low p27 levels (black curve). 


\section{Discussion}

$C D C 25 A$ behaves as an oncogene in murine fibroblasts. We thus investigated the expression of Cdc $25 \mathrm{~A}$ in a previously characterized series of human breast carcinomas, to assess whether its oncogenic potential in vitro is accompanied in vivo by overexpression in carcinomas, particularly in those tumors with more aggressive behavior. A cohort of patients with invasive carcinomas that measured less than $1 \mathrm{~cm}\left(\mathrm{~T}_{1 \mathrm{a}, \mathrm{b}}\right)$ was studied. Patients with these tumors represent up to $30 \%$ of patients presenting with invasive carcinoma and have a generally good prognosis (23), but a significant mortality of up to $13 \%$ is still recorded (18, $24)$. In the original cohort of patients analyzed $(n=$ 202), we had previously demonstrated that loss by tumor cells of the Cdk inhibitor p27, which acts predominantly to inhibit the cyclin E-Cdk2 complex, was a significant predictor of survival even in nodenegative patients (18). Here, using the residual samples available from this database, we have shown that overexpression of Cdc25A is also associated with poor prognosis, particularly in patients with tumors with low 27. Furthermore, it appears that stratification into four categories according to p27 and Cdc25A status separates survival curves by Cdc25A expression, suggesting that overexpression of the phosphatase may be a critical determinant of the biologic behavior in these tumors. Unfortunately, because of the limited number of cases examined and because of the relatively low overall mortality in this cohort of patients (13\%), multivariate analysis failed to demonstrate the independent prognostic significance of Cdc25A. However, the scope of this study was not to identify yet another prognostic marker in breast cancer, but to evaluate Cdc25A's role in breast cancer and to determine whether this phosphatase itself or its substrates constitute potential therapeutic targets in the early phases of this disease. Larger series of higher-stage breast cancers (with intrinsically higher mortality rates) will have to be analyzed to determine the independent prognostic significance of Cdc25A in breast carcinomas. In addition, development of antibodies that immunoreact in paraffin-embedded tissue (the ones we used did not, after exhaustive trials) will both validate the data presented and allow the analysis of larger databases.

We have shown that inactivation of CDC25A by antisense oligonucleotides inhibits S-phase entry in MCF-7 breast cancer cells. Although both S-phase fraction quantitation and Ki67 index have prognostic significance in breast cancer $(25,26)$, in our series of small breast cancers the proliferative index as assessed by Ki67 immunostaining was extremely low (18) and did not show significant correlation with either prognosis or Cdc25A levels. This lack of correlation may be ascribed to the low sensitivity of Ki67 immunohistochemistry in the determination of cell proliferation in the early stages of breast cancer, in which only a low percentage of tumor cells is cycling and a limited number of cells is available for assessment.

Our data suggest that both Cdc25A and its substrate $\mathrm{Cdk} 2$ represent important therapeutic targets in breast cancer. In fact, using microdissected breast carcinomas, we demonstrated that Cdc25A expression, together with cyclin E, correlated with Cdk2 enzymatic activity. In addition, oligonucleotides designed to inactivate $\mathrm{Cdc} 25 \mathrm{~A}$ resulted in inhibition of Cdk2 enzymatic activity and G1 arrest in MCF-7 cells. As expected, inhibition of Cdk2 was accompanied by increased tyrosine phosphorylation of Cdk2 itself. Our data obtained in breast cancer cells support initial (22) and recent (13) evidence obtained in other cellular systems that $\mathrm{Cdk} 2$ undergoes increased tyrosine dephosphorylation as cells enter S phase. The decrease in BrdU incorporation, which occurred as a result of antisense $C D C 25 A$ transfection, could be overridden by a Cdk2 mutant, which cannot be phosphorylated, but not by wild-type Cdk2. In contrast, inactivation of Cdc25A has no effect on Cdk4 activity, suggesting that $\mathrm{Cdk} 2$ complexes are specific targets of Cdc25A. Accordingly, overexpression of Cdc25A has been shown to lead to a premature activation of both cyclin E-Cdk2 and cyclin A-Cdk2 complexes, with no effect on cyclin D-dependent kinases (13). Finally, Cdc25A degradation has been shown to be the early DNA damage checkpoint mechanism resulting in a p53-independent blockage of S-phase entry via inhibition of Cdk2 activity (14, 15). Overexpression of Cdc $25 \mathrm{~A}$, which we find in $47 \%$ of $\mathrm{T}_{1 \mathrm{a}, \mathrm{b}}$ breast carcinomas, may thus contribute to overriding a physiologic G1 block in breast tumor cells. Taken together, these data strongly suggest that Cdk2 activation by Cdc25A is necessary for S-phase entry in human breast cancer.

Overexpression of Cdc25A has been shown to titrate p21 off of the cyclin E-Cdk2 complex, thus potentiating cyclin E-Cdk2 activity (21). Here, a decrease of p27 was found when antisense oligonucleotides against CDC25A were transfected in MCF-7 cells, suggesting that cyclin A-Cdk2 and cyclin E-Cdk2 complexes not activated by Cdc25A may not require inhibition by p27.

In summary, we have shown that Cdc25A activates Cdk2 and promotes S-phase entry in breast cancer cells. Cdc25A overexpression is associated with a worse outcome in patients with $\mathrm{T}_{1 \mathrm{a}, \mathrm{b}}$ breast carcinomas who undergo potential curative surgery. Our study suggests that adjuvant therapy, which benefits patients with advanced breast cancer, may also be appropriate for patients with $\mathrm{T}_{1 \mathrm{a}, \mathrm{b}}$ disease whose tumors have lost p27 and overexpress Cdc25A. Specific inhibitors of both Cdc25A and cyclin-dependent kinases such as $\mathrm{Cdk} 2$, should they became available, may prove to be particularly useful in this setting.

\section{Acknowledgments}

We thank Kinga Szigeti, Laura Tabatabai, Carmen Tam, Peter Worland, and Karen Juda for their contri- 
bution to this work; David Morgan for kindly providing the catalytic mutant for Cdk2 (Cdk2AF); Arthur $K$. Lee and Molly Warner for help with the databases and patient follow-up; Jane Hayward for assistance with the illustrations; and William Sellers, Myles Brown, and Marc Ewen for critical review of the manuscript. This study was supported by Department of Defense grant PC970273 and NIH grant RO1CA81755 to M. Loda, and Human Frontier Science Program Organization grant RG0229 and NIH grants R01-CA76584, R01-GM57587, P30-CA16087, and R21-CA66229 to M. Pagano.

1. Lees, E. 1995. Cyclin dependent kinase regulation. Curr. Opin. Cell Biol. 7:773-780.

2. Sherr, C.J., and Roberts, J.M. 1999. CDK inhibitors: positive and negative regulators of G1-phase progression. Genes Dev. 13:1501-1512.

3. DelSal, G., Loda, M., and Pagano, M. 1996. Cell cycle and cancer: critical events at the G1 restriction point. Crit. Rev. Oncog. 7:127-142.

4. Pardee, A.B. 1989. G1 events and regulation of cell proliferation. Science. 246:603-614.

5. Draetta, G., and Eckstein, J. 1997. Cdc25 protein phosphatases in cell proliferation. Biochim. Biophys. Acta. 1332:M53-M63.

6. Sadhu, K., Reed, S.I., Richardson, H., and Russell, P. 1990. Human homolog of fission yeast cdc25 mitotic inducer is predominantly expressed in G2. Proc. Natl. Acad. Sci. USA. 87:5139-5143.

7. Galaktionov, K., and Beach, D. 1991. Specific activation of cdc25 tyrosine phosphatases by B-type cyclins: evidence for multiple roles of mitotic cyclins. Cell. 67:1181-1194.

8. Gabrielli, B.G., Lee, M.S., Walker, D.H., Piwnica-Worms, H., and Maller, J.L. 1992. Cdc25 regulates the phosphorylation and activity of the Xenopus cdk2 protein kinase complex. J. Biol. Chem. 267:18040-18046.

9. Sebastian, B., Kakizuka, A., and Hunter, T. 1993. Cdc25M2 activation of cyclin-dependent kinases by dephosphorylation of threonine-14 and tyrosine-15. Proc. Natl. Acad. Sci. USA. 90:3521-3524.

10. Hoffmann, I., Draetta, G., and Karsenti, E. 1994. Activation of the phosphatase activity of human cdc25A by a cdk2-cyclin E dependent phosphorylation at the G1/S transition. EMBOJ. 13:4302-4310.
11. Lammer, C., et al. 1998. The cdc25B phosphatase is essential for the G2/M phase transition in human cells. J. Cell Sci. 111:2445-2453.

12. Jinno, S., et al. 1994. Cdc25A is a novel phosphatase functioning early in the cell cycle. EMBO J. 13:1549-1556.

13. Blomberg,I., and Hoffmann, I. 1999. Ectopic expression of Cdc25A accelerates the $\mathrm{G}(1) / \mathrm{S}$ transition and leads to premature activation of cyclin E- and cyclin A-dependent kinases. Mol. Cell. Biol. 19:6183-6194.

14. Mailand, N., et al. 2000. Rapid destruction of human Cdc25A in response to DNA damage. Science. 288:1425-1429.

15. Molinari, M., Mercurio, C., Dominguez, J. Goubin, F., and Draetta, F.G. 2000. Human cdc25 A inactivation in response to $\mathrm{S}$ phase inhibition and its role in preventing premature mitosis. EMBOJ. In press.

16. Galaktionov, K., et al. 1995. CDC25 phosphatases as potential human oncogenes. Science. 269:1575-1577.

17. Ma, Z.Q., Chua, S.S., DeMayo, F.J., and Tsai, S.Y. 1999. Induction of mammary gland hyperplasia in transgenic mice over-expressing human Cdc25B. Oncogene. 18:4564-4576.

18. Tan, P., et al. 1997. The cell cycle inhibitor p27 is an independent prognostic marker in small $(\mathrm{T} 1 \mathrm{a}, \mathrm{b})$ invasive breast carcinomas. Cancer Res. 57:1259-1263.

19. Magi-Galluzzi, C., et al. 1997. Mitogen-activated protein kinase phosphatase 1 is overexpressed in prostate cancers and is inversely related to apoptosis. Lab. Invest. 76:37-51.

20. Pagano, M., et al. 1995. Role of the ubiquitin-proteasome pathway in regulating abundance of the cyclin-dependent kinase inhibitor p27. Science. 269:682-685.

21. Saha, P., Eichbaum, Q., Silberman, E.D., Mayer, B.J., and Dutta, A. 1997. p21CIP1 and Cdc25A: competition between an inhibitor and an activator of cyclin-dependent kinases. Mol. Cell. Biol. 17:4338-4345.

22. Gu, Y., Rosenblatt, J., and Morgan, D.O. 1992. Cell cycle regulation of CDK2 activity by phosphorylation of Thr160 and Tyr15. EMBO J. 11:3995-4005.

23. Cady, B., et al. 1996. The new era in breast cancer. Invasion, size, and nodal involvement dramatically decreasing as a result of mammographic screening. Arch. Surg. 131:301-308.

24. Rosen, P.P., Groshen, S., Kinne, D.W., and Norton, L. 1993. Factors influencing prognosis in node-negative breast carcinoma: analysis of 767 T1N0M0/T2N0M0 patients with long-term follow-up. J. Clin. Oncol. 11:2090-2100.

25. Wenger, C.R., and Clark, G.M. 1998. S-phase fraction and breast cancer: a decade of experience. Breast Cancer Res. Treat. 51:255-265.

26. Dettmar, P., et al. 1997. Prognostic impact of proliferation-associated factors MIB1 (Ki-67) and S-phase in node-negative breast cancer. Br. J. Cancer. 75:1525-1533. 